\title{
Entrelacs
}

Cinéma et audiovisuel

13 | 2017

Espace, perspective et fragmentation

\section{Le Matte Painting : Du trompe-l'œil au trompe la perspective}

Réjane Hamus-Vallée

\section{OpenEdition}

1 Journals

Édition électronique

URL : http://journals.openedition.org/entrelacs/2051

DOI : 10.4000/entrelacs.2051

ISSN : 2261-5482

Éditeur

Éditions Téraèdre

Référence électronique

Réjane Hamus-Vallée, "Le Matte Painting : Du trompe-l'œil au trompe la perspective », Entrelacs [En ligne], 13 | 2017, mis en ligne le 05 mars 2017, consulté le 10 décembre 2020. URL : http:// journals.openedition.org/entrelacs/2051; DOI : https://doi.org/10.4000/entrelacs.2051

Ce document a été généré automatiquement le 10 décembre 2020.

Tous droits réservés 


\title{
Le Matte Painting : Du trompe-l'œil au trompe la perspective
}

\author{
Réjane Hamus-Vallée
}

1 Le Matte Painting consiste à combiner dans un même plan large une scène réelle et son extension réalisée par peinture. La composition d'un élément issu d'une prise de vues «réelle», "directe» (live pour reprendre le terme anglo-saxon) avec une peinture a connu tout au long de l'histoire du cinéma des techniques d'exécution très différentes, dont rend compte la richesse du vocabulaire anglo-saxon sur la question. Le «glass shot », littéralement " plan de verre ", fut la première technique employée. Elle consiste simplement à suspendre entre le décor et la caméra une "vitre ", sur laquelle le peintre vient rajouter les éléments nécessaires. Puis vient ensuite le matte painting ${ }^{1}$, " peinture de cache ", qui consiste à filmer le décor réel, et à rajouter la peinture en post-production, grâce à un cache noir (matte en anglais), et son contre-cache complémentaire. Le digital matte painting, peinture cache numérique, vient achever, à ce jour, les transformations technologiques qu'a connues ce principe de base (combiner ensemble peinture et portion de l'espace réel) ${ }^{2}$.

2 Ce trucage cinématographique connaîtra des utilisations totalement opposées, qui vont de l'effet invisible mais perceptible, à l'effet imperceptible, si l'on reprend la distinction de Christian Metz ${ }^{3}$. Dans le cas de l'effet « invisible mais perceptible», le spectateur sait qu'il y a trucage grâce au contexte (espace imaginaire, fantaisiste) et à ses connaissances. Mais ce qui est invisible pour lui, c'est la technique qui lui a donné naissance - le trucage est donc invisible, mais son effet est perceptible. A l'inverse, les trucages imperceptibles (un plafond de salle de bal par exemple) ne sont pas soupçonnés par le spectateur, qui ne peut donc ni voir la technique de trucage, ni ressentir un effet « spécial ».

3 Dans les deux types d'effets, l'objectif recherché n'est atteint que si la peinture, cause technique, ne se voit jamais en tant que telle. Un plan avec un Matte Painting repose sur un effacement généralisé de sa nature profilmique - ici la peinture - pour obtenir un effet diégétique - la création d'un large espace. Toute l'histoire du Matte Painting expose une palette sans cesse renouvelée de "trucs et astuces » (" gags » destinés à effacer l'origine picturale du trucage) : un plan de matte est donc en permanence un plan où luttent d'un 
côté les détails illusionnistes, éléments précis dans l'image cherchant à authentifier, par contagion, le reste du plan (oiseaux qui volent, nuages qui se déplacent, spectateurs qui applaudissent...) et des détails désillusionnistes, qui révèlent clairement, si le spectateur les perçoit, la nature truquée du plan: aplats de couleur imprécis, acteur dont la tête disparaît derrière la ligne de cache qui sépare la peinture sur décor réel, ou encore perspective visiblement fragmentée.

4 La perspective dans ce cadre est donc un facteur essentiel pour comprendre ce trucage, au moins à trois niveaux

D'une part, l'obligation d'avoir une perspective unique contraint les matte painters à reproduire scrupuleusement les lignes de fuite déjà présentes dans l'image cinématographique, référence absolue de leur travail pictural. La difficulté provient de la nécessité de construire une seule perspective globale, à partir de deux fragments d'espace : seule la caméra relie et unifie ces deux fragments, et la moindre variation par rapport à ce point de vue unique expose clairement l'artifice. Une ligne brisée révèlerait immédiatement la nature double de l'image, et annihilerait toute forme d'illusion. La perspective linéaire centrale est un facteur qui autorise l'équation : 1+1 =1 (l'image du tournage plus son extension en peinture égale un plan unique, homogène).

D'autre part, l'effet perspective est indispensable pour créer un effet relief, essentiel pour effacer l'objet peinture (en « 2D ») dans l'esprit du spectateur.

Enfin, de par sa nature d'extension de décors et de plans larges, le matte painting introduit nécessairement des lignes de fuite prolongées, ouvrant l'espace, et donc là aussi jouant de la notion de perspective pour accroitre le champ et plonger le regard du spectateur dans un abîme parfois sans fin.

8 La perspective est donc le premier détail illusionniste nécessaire pour provoquer la sensation de "vrai» dans ce type de plan. La maîtrise des règles et outils de la perspective renaissante est donc primordiale pour les peintres sur verre : la construction des peintures repose sur les lignes de force calquées depuis le plan filmé en live, et l'emploi de règles et d'appareil proches, par exemple, du portillon de Dürer comme le montre un extrait du brevet ( $n^{\circ} 1.372 .811$ déposé le 23 décembre 1918, délivré le 29 mars 1921) de Walter G. Hall pour son procédé éponyme, très utilisé en France et aux USA au cours des années 1920.
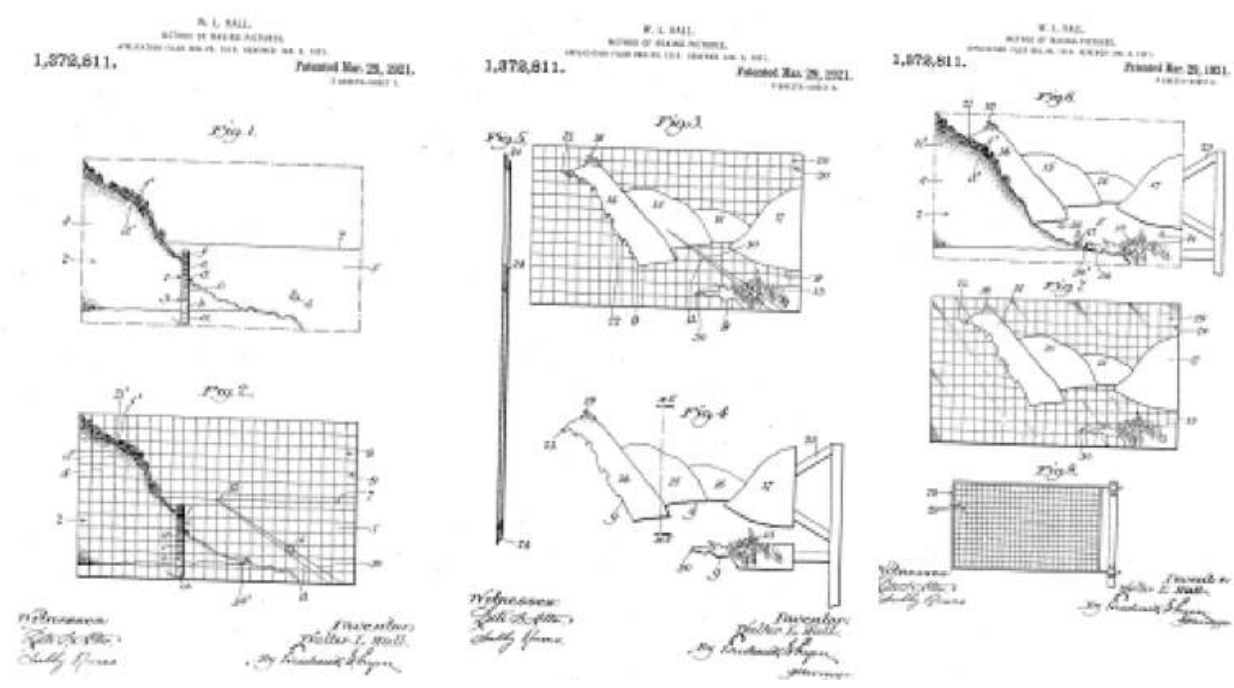

Extrait du brevet de Hall, exposant les différentes étapes et les accessoires nécessaires pour pouvoir prévoir la miniature, et la fabriquer sur du contre-plaqué4. 
Sur les photos montrant les matte painters travailler sur leurs vitres, les lignes de force sont tracées et prolongées dans la peinture ${ }^{5}$. C'est aussi ce que les technologies numériques ont exploré avec des analogies très fortes entre les peintres renaissants, et les premiers tests d'images numériques. Le matte painter Chris Evans, passé de 1 'analogique au numérique, rappelle qu'il vient :

\begin{abstract}
de la peinture renaissante, c'était donc intéressant de voir que l'amour et l' habileté développés pour la peinture traditionnelle m'ont amené à de la haute technologie en étant le premier artiste du film à travailler sur un ordinateur. Ce qui était passionnant avec les développeurs d'images de synthèse était qu'ils avaient affaire avec beaucoup d'éléments essentiels déjà rencontrés par les artistes de la Renaissance - définir des cubes et des sphères et des cylindres, la couleur, la lumière des objets, les textures. Toutes ces choses déjà étudiées par les artistes de la Renaissance ont été transposées dans le monde mathématique de l'ordinateur. ${ }^{6}$
\end{abstract}

10 Ce qui explique que de nombreux matte painters sont d'abord et avant tout des peintres mais que cette seule condition ne suffit pas à en faire des spécialistes du trucage. Les entretiens avec les professionnels reviennent régulièrement sur des anecdotes exposant à quel point les peintres ont du mal à se faire aux contraintes cinématographiques : ne pas faire une belle peinture en soi, mais une belle peinture cinématographique... «"Etre un bon matte artiste", pense Whitlock, "nécessite une très bonne connaissance de la photographie et de ses limites, et vous devez vous rendre compte que vous ne faites pas vraiment une peinture en soi... Quand vous créez un matte qui possède une fenêtre, une zone noire, pour l'action, c'est très facile de créer simplement une peinture merveilleuse en oubliant totalement l'action qui est censée s'intégrer à l'intérieur. Au final, quand la peinture est achevée et que l'action y est insérée, vous ne pouvez plus ôter vos yeux de cette belle peinture, et vous ratez totalement l'action." " "

11 Nous voudrions donc ici questionner le mode de fonctionnement de ce trucage cinématographique, en partant dans un premier temps de ce qui semble, à première vue, son ancêtre direct, le trompe-l'œil, grand utilisateur aussi de perspective linéaire, pour voir comment, en passant au cinéma, ce trompe-l'œil devient un trompe la caméra - et en particulier, un trompe la perspective. Nous analyserons dans ce second temps deux usages opposés de la perspective, son usage majeur illusionniste à travers le travail du peintre Albert Whitlock en particulier pour ses trucages de film hitchcockiens, et un usage désillusionniste, un des rares exemples de perspective volontairement fragmentée pour le film d'Alain Resnais, La vie est un roman de 1983, et les peintures de Jean-Marie Vives d'après Enki Bilal. Par son anormalité, ce dernier exemple expose clairement la norme qui nous préoccupe ici, et l'usage dominant de la perspective comme outil organisant la direction de regard du spectateur, et donc organisant l'illusion à l'œuvre dans ces plans truqués. Par l'exception qu'il apporte, cet exemple offre une mise en abîme du dispositif, qui lui-même peut se comprendre comme une mise en abîme du dispositif illusionniste du cinéma en son entier.

\title{
Le matte painting, héritier du trompe-l'œil ?
}

« La définition du trompe-l'œil est apparemment simple : c'est une façon de peindre quelque chose de manière que cette chose ait l'air non peinte, 
mais vraie; ou, si l'on préfère, c'est une peinture qui s'efforce d'imiter à s'y méprendre le réel. ${ }^{8}$ " trompe-l'œil. Les rares articles théoriques citant le Matte Painting l'assimilent régulièrement avec ce «trompe-l'œil ». Ainsi, Pascal Bonitzer se demande dans quelle mesure « le trompe-l'œil serait-il le dénominateur commun du cinéma et de la peinture ? Du plan et du tableau ? $^{9}$ \%. Le Matte Painting fait-il partie de la longue famille des trompel'œil picturaux, ou cette assimilation relève-t-elle d'un abus de langage? En quoi l'objet «peinture sur verre » se rapproche-t-il, ou non, des trompe-l'œil « classiques»? Nous verrons dans un premier temps les points communs entre le trompe-l'œil et le Matte Painting, mais aussi leurs différences fondamentales.

Premier point commun : la notion de relief. Pour arriver à l'effet décrit par Perec, le trompe-l'œil se doit de camoufler sa nature première d'image à deux dimensions, pour devenir un (ou des) objet(s) en trois dimensions dans le regard du spectateur. D'où l'importance de la perspective pour créer cet effet relief, et passer de la 3D (objet réel) à la 2D (peinture) pour arriver à la 3D (objet réel) dans l'esprit du spectateur.

Deuxième point : le point de vue. Cet effet relief est totalement dépendant d'un dispositif de perception visuelle créé autour de l'œuvre. La base de ce dispositif consiste évidemment à prendre en compte l'œil à tromper, soit le regard du spectateur. Que l'on soit placé à quelques centimètres de l'œuvre, ou à plusieurs mètres, l'effet ne sera pas le même. Conséquence, le trompe-l'œil est conçu pour un point de vue particulier. C'est d'ailleurs par changement de points de vue que l'œil se détrompe souvent : lorsqu'on s'en approche, la peinture reprend ses droits sur la sensation de relief suggérée par la perspective peinte. Lorsque le spectateur est placé au point de vue «spécial », se produit alors l'effet «spécial ». C'est la rencontre d'une peinture et d'un regard au sein d'un dispositif précis qui crée cet effet - la peinture seule, déconnectée du dispositif, n'est pas assurée de tromper l'œil.

Troisième point: l'effacement. Ce dispositif passe par un nécessaire effacement des frontières du tableau ${ }^{10}$, et parfois même du cadre qui peut révéler au spectateur la nature de l'objet qu'il observe. Au cours de l'histoire du trompe-l'œil, ces frontières entre réel et peinture ont été dépassées de différentes façons : soit le cadre est partie intégrante du trompe-l'œil, soit il disparaît totalement, plaçant la peinture à l'endroit de l'objet repr ésenté, tel le violon suspendu derrière une porte de l'œuvre Violon peint, attribuée à Jan Van der Vart, début du $18^{\text {ème }}$ siècle. Le "réel» doit transformer la peinture, qui inversement le prolonge et le transforme à son tour. Cette notion de frontière est encore plus flagrante dans le cas de trompe-l'œil monumentaux, plafonds d'église, fausses galeries, fausses fenêtres. C'est aussi le cas dans la fameuse compétition Zeuxis vs Parrhasios telle que racontée par Pline, avec le rideau peint trompant l'artiste. «On raconte que ce dernier [Parrhasios] entra en compétition avec Zeuxis: celui-ci avait présenté des raisins si aisément reproduits que les oiseaux vinrent voleter auprès d'eux sur la scène ; mais l'autre présenta un rideau peint avec une telle perfection que Zeuxis, tout gonflé d'orgueil à cause du jugement des oiseaux, demanda qu'on se décidât à enlever le rideau pour montrer la peinture, puis, ayant compris son erreur, il céda la palme à son rival avec une modestie pleine de franchise, car, s'il avait personnellement, disait-il, trompé les oiseaux, Parrhasios l'avait trompé lui, un artiste. ${ }^{11}$ » 

aptes à tromper l'œil du spectateur : la mouche est ainsi un grand classique des trompel'œil. Car si tel n'était pas le cas, le côté extraordinaire de l'objet représenté le révèlerait aussitôt au public comme digne d'attention, et donc, dévoilerait plus facilement l'illusion que dans le cas déjà cité du violon suspendu à sa place, « comme si de rien n'était », derriè re la porte. Dans le même ordre d'idée, «les objets représentés et sélectionnés par l'artiste réalisant un trompe-l'œil doivent appartenir au registre des choses muettes, des êtres inanimés..$^{13}$ " A ce titre, les personnages vivants "sont à éviter ", non seulement pour des soucis de réalisme, mais surtout parce que leur apparence se démode vite, rendant visible immédiatement le trompe-l'œi ${ }^{14}$. Le temps de l'illusion est forcément beaucoup plus court avec un personnage vivant, le manque anormal de mouvement venant immédiatement exposer sa nature picturale.

Cinquième point : l'argent. De nombreux trompe-l'œil, plus monumentaux cette fois-ci, sont créés pour économiser sur des coûts de construction : plafond bas, qui semble plus élevé grâce au trompe-l'œil, pièce agrandie par une fausse fenêtre...

Sixième point : l'exception. Le trompe-l'œil est toujours de l'ordre de l'exception, de la rareté, qui pousse au plus loin l'essence même de la peinture : après tout, la peinture en soi est une forme de trompe-l'œil. De fait, les écrits théoriques consacrés au trompe-l'œil sont souvent partagés entre l'idée que cette forme d'œuvre incarne l'essence même de la peinture, et de l'autre, qu'elle n'est qu'un simple exercice technique dépourvu de style, de signature et donc d'art. Dans le premier cas, le trompe-l'œil, en questionnant la place du spectateur, son rapport à l'œuvre, entraîne de fait une réflexion sur la notion de représentation, et sur l'ontologie de la peinture en son entier. Le trompe-l'œil en tant qu' ' exception » permet, par son caractère hors norme, de questionner «la norme ». En effet, "s'il est une véritable expérience sensible et optique, il est également une authentique épreuve intellectuelle. L'impression trouble et fugace qu'il suscite laisse une trainée comparable à celle d'une étoile filante dont la sidération et la subsistance provoqueront une réflexion sur l'illusion, ses causes et ses perversions..$^{15}$ "

Relief, point de vue, effacement, côté banal, coût et question de l'exception: autant d'éléments que l'on retrouve à l'identique dans le Matte Painting cinématographique, et qui le relient donc à son ancêtre. Mais au-delà de ces points communs subsiste une différence fondamentale : le trompe-l'œil fonctionne systématiquement en deux temps, un temps de tromperie, un temps où le spectateur se rend compte qu'il a été trompé. «Le trompe-l'œil - si la chose existe -, ou du moins le scénario du spectateur trompé, perd tout intérêt si l'impostura n'est pas dévoilée à un certain moment. ${ }^{16} »$ Pire, selon Milman, si ce dévoilement n'a pas lieu, «alors le trompe-l'œil reste une supercherie, un procédé, un dé cor vide de sens. ${ }^{17}$ » Si l'œil est trompé en permanence, il perçoit donc un objet, et pas le tableau trompe-l'œil. Il ne peut donc ni goûter la virtuosité de l'artiste, ni questionner les propres méandres de sa perception (puisque pas de désillusion suivant l'illusion). Or, le plaisir pris à la vision du trompe-l'œil provient aussi de cette virtuosité, et de la nécessaire comparaison entre l'objet et sa représentation si fidèle qu'elle se confond, $\grave{a}$ première vue, avec lui ${ }^{18}$. Un trompe-l'œil qui tromperait effectivement l'œil ne serait plus un trompe-l'œil... Autrement dit, le trompe-l'œil serait de l'ordre de la simulation, et non de l'illusion, si l'on suit Calabrese ${ }^{19}$. Dans tous les cas, il ne serait jamais trompeur. «En conclusion nous devrions définir le trompe-l'œil non pas comme une tromperie de la perception, mais comme une expression visuelle paradoxale. En effet, si son efficacité passe par l'habileté de l'artiste à tirer le meilleur parti des ressources techniques de la 
perspective, il est aussi vrai que cette même habileté finit par se confondre complètement avec l'image produite. Le trompe-l'œil fait voir, alors même qu'il dissimule. ${ }^{20}$ » Il faut que le spectateur finisse par voir la peinture, dissimulée au premier abord, pour que l'effet se produise.

La révélation spectatorielle apparaît comme le point central de divergence avec le Matte Painting cinématographique. Ici, le public ne peut se décaler sur le côté pour apercevoir le manque de relief du trompe-l'œil qui lui est proposé. Il ne peut que se douter, dans les cas de mondes extraordinaires par exemple, qu'il est effectivement trompé sur la nature de ce qui lui est montré. La ville du futur qu'on lui présente sur une vaste étendue n'existe pas, il le sait, mais il ne sait pas forcément comment l'image a été obtenue, maquette, peinture, décor à échelle, ou plus récemment image de synthèse. Et dans une large majorité de cas, le spectateur ne peut même pas imaginer qu'il a été trompé plafonds de salles de bal par exemple. Mais dans les deux cas, le spectateur cinématographique ne doit JAMAIS voir la peinture, sauf à rater totalement l'effet spécial. Un trompe-l'œil pictural est toujours de l'ordre de l'exposition, un trompe-l'œil cin ématographique toujours du camouflage. Certes, on pourrait évoquer le making of comme second temps du Matte Painting : on voit le film sans savoir qu'il est truqué, et on lit des éléments qui, ensuite, révèlent la supercherie. Mais c'est une vision très récente, d'autant que le plus souvent, le making of ou les entretiens de techniciens spécialisés se focalisent sur les effets spectaculaires, et non sur le plus gros travail du Matte Painting, à savoir les effets imperceptibles.

Si le Matte Painting trompe bien un œil, c'est celui de la caméra, non celui du spectateur : la caméra, elle, pourrait se décaler et révéler la supercherie ${ }^{21}$. C'est pour son point de vue que la peinture est créée, point de vue qui sera de fait celui du spectateur, contraint d'épouser la place qu'on lui attribue. C'est par rapport à la transformation de la caméra, aux distorsions créées par sa médiation, que le Matte Painting est pensé. Ce qui explique que les principes de base du Matte Painting soient très proches, au final, de "l'hyperréalisme » du trompe-l'œil, mais que sa mise en place aboutisse à des effets totalement opposés. Le Matte Painting cinématographique est donc plus un «trompe la caméra » qu'un trompe-l'œil au sens classique du terme - et donc en particulier, un trompe la perspective cinématographique ${ }^{22}$.

\section{Le trompe la perspective parfait, le travail d'Albert Whitlock dans les films d'Alfred Hitchcock}

Albert Whitlock est un des plus célèbres matte painters de l'histoire du cinéma. Commençant sa carrière à l'âge de 14 ans en Grande-Bretagne en 1929, il y rencontre Alfred Hitchcock, avec lequel il collabore aussitôt. Après un passage chez Walt Disney qui le fait venir au début des années 1950 aux USA, Whitlock prend la tête du département des mattes du studio Universal en 1961, jusqu'à sa retraite (et la fermeture dudit dé partement) en 1985. Il y collabore à nouveau avec Hitchcock pour ses films américains comme par exemple Les oiseaux en 1963, Pas de printemps pour Marnie en 1964, Le rideau déchiré en 1965, L'étau en 1969 ou Frenzy en 1972.

Whitlock est aussi connu pour avoir voulu expliquer son travail auprès du grand public et pour revendiquer une filiation directe avec l'impressionnisme, style venant directement du manque de temps rencontré par le peintre pour réaliser son travail ${ }^{23}$. «Comme dans 
l'Impressionnisme, mes peintures ne sont pas concernées par l'objet, mais par l'effet de lumière sur l'objet. (...) La lumière, le moment de la journée et les effets dans cette peinture sont plus importants que les constructions individuelles dont personne ne tiendrait compte de toute façon. ${ }^{24}$ » Autrement dit, les peintures de Whitlock sont composées de zones d'image floues, donnant une impression de lumière et de mouvement, et de zones très précises, vers lesquelles Whitlock guide notre attention, que ce soit via le son, la composition, le cadrage, le mouvement des acteurs et des détails secondaires (nuages, oiseaux justement). Whitlock peint d'abord les détails illusionnistes de son œuvre, puis les relie entre eux par des traits moins précis.

Parmi toutes les techniques de trucage qu'il a utilisées, Hitchcock est surtout célèbre pour son usage de la transparence ${ }^{25}$. Lui permettant de tourner en studio, et d'avoir un contrôle accru - car immédiat - sur le résultat final, la transparence est présente dans quasiment tous les films du réalisateur. Il en va de même du Matte Painting, qu'Hitchcock utilise aussi dans tous ses films, mais dans des plans plus courts, et surtout dans moins de plans au total que la transparence. L'usage qu'en fait Hitchcock se partage entre plans imperceptibles (la maison en haut de la colline de L'étau, toute la séquence dans le musée est-berlinois du rideau déchiré26, ou encore l'Albert Hall de L'homme qui en savait trop de 1934), et plans invisibles mais perceptibles (l'attaque climax de la station essence des Oiseaux, le final sur la statue de la Liberté de La cinquième colonne, ou le climax de Sueurs froides dans le clocher ou de La mort aux trousses sur les monts Rushmore).

Ainsi, les plans de Frenzy en Matte Painting sont totalement imperceptibles. Sans le making of, ce sont d'ailleurs des plans impossibles à distinguer des autres. Parmi eux, le plan en prison, dans une séquence très courte, expose la blessure volontaire de l'innocent condamné à tort, prélude à son évasion. Tout au fond de ce plan à gauche, à moitié mis en valeur par la profondeur de la perspective et à moitié cachés par la couleur verte des murs, se tiennent deux gardiens immobiles. Ces deux gardiens sont peints, figurants créant une échelle de taille, bien qu'il soit totalement impossible au spectateur de les voir clairement puisque le plan dure trois secondes. Mais clairement, les lignes de fuite entraînent notre regard vers le fond du plan, vers ces deux figurants immobiles, qui créent aussi, par leur présence corporelle, une échelle et une référence pour l'espace de la prison.

Ce plan de matte est intercalé entre un plan d'extérieur de la prison et de sa porte fermée (d'une durée de 3 secondes), qui sert à préciser au spectateur où il va se trouver dans le plan suivant (extérieur incluant l'intérieur dans une parfaite synecdoque), et un gros plan très rapide (2 secondes) sur le visage de l'innocent. Dans le plan de matte, l'innocent est déjà visible, ou plutôt audible grâce au son de ses pas et de celui de ses gardiens qui les signale à notre vue. Puis un plan rapproché sur l'escalier expose la chute, validée par un gros plan sur le visage de l'accidenté ensanglanté. La scène se finit en boucle, à l'extérieur de la prison, une ambulance sortant cette fois par la porte. Pour 30 secondes de plan, il y a donc un décor construit (l'escalier) et un Matte Painting exposé trois secondes : juste assez pour percevoir que les couloirs de la prison sont déserts (c'est le soir), grands (et donc, le moindre personnage est fort visible), signifiant alors la difficult é voire l'impossibilité de s'échapper (les gardiens sont partout). Mais ces trois secondes ne laissent pas assez de temps pour voir que les gardiens sont immobiles, et qu'il n'y a d'ailleurs aucun mouvement dans le plan, hormis celui des protagonistes principaux. Dans cette rapidité volontaire, la forte perspective est indispensable, à la fois pour donner des clés très rapides de compréhension de cet espace et pour renforcer l'illusion en 
organisant un mouvement des yeux du spectateur durant ces trois secondes, durée standard de montage des plans avec Matte Painting (entre 3 à 5 secondes).

Inversement, l'autre plan de Matte Painting du film ${ }^{27}$ (la vue de la rue depuis l'appartement du tueur) est monté totalement différemment. Ce plan intervient au moment où le spectateur devine que le personnage a à nouveau assassiné une femme, et suppose donc qu'il va se débarrasser du corps. Par conséquent, le suspense est ici à son comble : le meurtrier va-t-il réussir à se débarrasser du corps sans être inquiété ?

On le voit sortir de chez lui, en plan rapproché, sur son perron, poussant un chariot sur lequel est posé un gros sac: le cadavre. Le plan suivant est la vue «depuis la fenêtre de son appartement ", qui le montre en train de pousser le chariot dans la rue vide. Il dure 16 secondes, ce qui est très long pour un Matte Painting. Mais ces 16 secondes sont essentielles pour faire durer le suspense, puisque c'est le temps nécessaire pour que le personnage accomplisse le trajet de sa porte au camion. Durant ce trajet, le spectateur est focalisé sur le personnage (seul élément éclairé du plan, seul lieu en mouvement, bruit de pas), tout en étant attiré par l'univers sombre qui l'entoure, aidé en cela par les perspectives clairement déformées de Whitlock: quelqu'un va-t-il surgir de cette obscurité pour arrêter le meurtrier? Par conséquent, Hitchcock détourne une règle classique de montage (cinq secondes maximum pour un plan avec Matte Painting), pour au contraire insister sur l'immobilisme de la ville la nuit, ce qui permet en retour de crédibiliser le Matte Painting et de tirer avantage de ses contraintes (il est logique que rien ne bouge vue l'heure tardive). Puisque le spectateur cherche et espère du mouvement, il n'en trouvera aucun. Albert Whitlock n'utilise aucun de ses « gags » favoris ici - d'autant que l'obscurité lui permet de camoufler la peinture. Whitlock joue ainsi sur la frustration et l'incompréhension visuelle du spectateur qui se trouve face à une mise en scène originale de l'action et du suspense, ce qui permet d'augmenter, de fait, la tension dramatique. Dans ce cas particulier, une durée trop courte ne permettrait pas de créer l'angoisse paradoxale à laquelle est soumis le spectateur : peur de voir le personnage se faire prendre et peur qu'il ne se fasse pas prendre. Les fortes perspectives du plan reprennent ce paradoxe: d'une part, force centrifuge, elles orientent notre regard vers les bords - les possibles témoins - d'autre part, au centre du plan, force centripète, elles sont redoublées par le mouvement en biais du personnage, qui suit scrupuleusement la ligne de force vers la gauche du plan, nous forçant à suivre son macabre cheminement. Encore une fois, la perspective est utilisée ici pour aider à la compréhension du plan, et ici, à sa tension interne.

\section{L'exception qui confirme la règle, le travail de Jean- Marie Vives et d'Alain Resnais pour La vie est un roman}

La vie est un roman d'Alain Resnais en 1983 propose de ne conserver que la peinture exhibée, et d'utiliser le glass shot pour ce qu'il est : une couche de peinture hétérogène, intercalée entre la caméra et l'acteur. Ce contre-exemple dans l'utilisation des "glass paintings " est évidemment un cas d'école : rares sont les films qui exhibent ainsi la peinture comme peinture, sans chercher à effacer la cause. Dans ce cas bien particulier, la cause même devient l'effet... Et c'est cette particularité qui va nous permettre, a contrario, d'interroger le Matte Painting traditionnel - celui à base de camouflage de la peinture et de perspective unique. 
30 Cette volonté très forte d'exhibition de la part de Resnais sera ainsi en permanence rappelée à Jean-Marie Vives, en charge des glass shots utilisés sur le film. Réalisées d 'après les dessins de l'illustrateur Enki Bilal, les peintures ne devaient pas être "trop " intégrées à l'univers onirique présenté derrière elles. Il s'agissait d'avoir ici une image cinéma ET une image dessinée, les deux restant volontairement hétérogènes. Ainsi que l'explique le matte painter Jean-Marie Vives, «je photographiais le dessin qu'Enki me soumettait et je l'adaptais sur la vitre. Ensuite venait le dessin en couleurs où, sur le glass painting, je devais redonner du volume afin qu'il se raccorde le mieux possible à la prise de vue. Ma tâche se trouvait extrêmement simplifiée dans la mesure où je n'avais aucun raccord à faire entre le dessin de Bilal et le décor réel. Il fallait absolument gommer le côté hyperréaliste du procédé pour bien montrer qu'il s'agissait d'une peinture. ${ }^{28}$ »

31 Après un premier test qui séduit Alain Resnais, Jean-Marie Vives exécute les glass shots du film "au préalable», et non sur place, méthode incompatible avec une peinture traditionnelle à perspective unique. Peu importe alors si les raccords de perspective ne sont pas parfaits, ou si les acteurs sont ensuite limités dans leurs mouvements par les zones peintes, ce qui est à l'encontre du travail habituel du peintre Jean-Marie Vives, qu'il le réalise avec un pinceau ou une palette graphique. "Quand je faisais des peintures traditionnelles, je faisais très attention à ce que je faisais autour du personnage, et beaucoup moins à ce qu'il y avait dans le coin en haut à gauche... Même en numérique, je continue à laisser la lumière sur les personnages, et toutes les perspectives fuient vers eux. J'essaie d'obliger les gens à regarder un point et pas un autre. ${ }^{29}$ »

32 Les peintures interviennent à des moments bien précis du film, lorsque le château tout entier semble en proie aux rêves. Le film s'articule autour de trois espace-temps qu'il mélange, le château à ses débuts après la Première Guerre mondiale, dans lequel se déroule "l'expérience de Forbeck »; le château à l'époque contemporaine, soit 1982, qui accueille un colloque sur l'éducation de l'imagination; et enfin les «temps légendaires », espace-temps indéfini, qui accueille tous les glass paintings du film.

Le premier glass painting du film apparaît à la $7^{\text {ème }}$ minute. Plus la nourrice s'enfonce dans la forêt à droite, plus la peinture apparaît, révélée par un panoramique latéral qui suit le trajet de la femme. Ce mouvement est tout à fait révélateur du mouvement général du film : du « réel » à l'imaginaire, les deux étant absolument liés via la continuité créée par le même mouvement, dans le même espace.

34 Ce plan nous donne aussi les clés pour comprendre l'utilisation du glass painting dans le film. La peinture est très visible, totalement hors raccord avec la partie « réelle » du plan, mais le dispositif en lui-même reste camouflé. Par exemple, le montant latéral nécessaire pour tenir la peinture a été caché par un arbre. De même, la caméra sera systématiquement recouverte de noir (borniolée), seul un trou étant laissé pour l'objectif, afin d'éviter tout reflet sur la vitre, qui exposerait ainsi le dispositif. On est donc ici en permanence dans une approche mixte : mixte de végétation réelle et artificielle, et à un autre niveau de lecture, mixte d'exposition du procédé et de camouflage.

Cette fin de plan représente alors un vrai défi pour le spectateur, qui reçoit deux stimuli opposés : doit-il regarder le premier plan et chercher à comprendre ce qu'il représente, ou rester focalisé sur la femme qui disparaît, au fond, faisant ainsi le point sur un deuxième plan plus lointain? Car la profondeur qu'apporte ici la peinture est contradictoire avec les règles perspectivistes habituelles, et apporte un élément perturbateur (une texture et une couleur différentes entre ce premier plan et le fond) au 
spectateur qui n'a pas l'habitude d'avoir ce type de plan face à lui. «En effet, suivant le souhait d'Alain Resnais, il n'y avait pas de raccords perspective ou chromatique afin de placer les glass paintings dans une ambiance vraiment bande dessinée. ${ }^{30}$ "

Malgré tout, le mouvement de caméra fut ici réalisé avec un pied nodal, qui permet d'effectuer des panoramiques verticaux et latéraux sans changement de perspective: pourquoi utiliser ce dispositif "illusionniste", particulièrement complexe, puisque l'enjeu du plan est justement de défaire les règles habituelles de perspective unique ? Se défaire de la perceptive linéaire est donc difficile pour le spectateur, pour qui l'œil et le cerveau entrent en opposition, tant il cherche malgré tout à raccorder l'irraccordable, tout comme cela reste difficile pour l'équipe du film. Car en dépit de la volonté de Resnais, la perspective linéaire apparaît parfois plutôt respectée, en particulier dans la salle du trône, que l'on voit à deux reprises, avec davantage de colonnes la deuxième fois. C'est ce que confirme Jean-Marie Vives ${ }^{31}$, qui explique que ces plans étaient les moins appréciés de Resnais, car «trop raccord ». De même, pour le plan situé à l'intérieur de la grotte, les acteurs devaient dans un premier temps venir vers la caméra, entre les colonnes peintes. Mais le changement de décor et les essais prouvent que ce mouvement les fait «trop " grandir dans l'œil de la caméra. Les acteurs se mettaient à déborder du chemin peint, et la peinture devenait ici visible en étant de taille trop différente de celle des acteurs. Le mouvement final des acteurs fut alors latéral, comme tous les autres mouvements du film, accentuant davantage la confrontation entre deux plans de vision : le premier plan, plat - celui de la peinture -, le second plan - celui des acteurs -, lui aussi globalement perpendiculaire à l'axe de la caméra. Les deux plans sont donc parallèles entre eux, deux univers irrejoignables.

37 A l'inverse de ce que prône le matte painter Chris Evans, pour qui le Matte Painting est une « fenêtre ouverte sur une vue ${ }^{32}$ » nécessitant une absence de sens de la surface, le plat et la surface sont omniprésents ici. La vision du spectateur est donc prise entre deux « anomalies ». D'une part, la contradiction évidente entre les acteurs de chair et d'os, la forêt, et la peinture plate. D'autre part, la contradiction entre des éléments communs entre ces deux espaces (éléments figuratifs, colonnes) qui les relient, et des éléments totalement différents (couleurs, perspective) qui les séparent. C'est une mise en évidence de la «double réalité » de l'image, telle que décrite par Jacques Aumont à propos de la peinture, mais plus largement caractéristique de toute image. « Double réalité, puisque l'œ il perçoit en même temps l'espace plat de la surface de la toile et la vue partielle sur un fragment d'espace "en profondeur", produite entre autres par l'emploi de la perspective. ${ }^{33}$ " C'est cette contradiction profonde entre le plat et le relief que met en abyme cet usage du Matte Painting dans La vie est un roman, provoquant ce malaise du spectateur.

La vie est un roman met donc en avant le non-respect des perspectives, des lumières et des textures; la peinture comme bande dessinée et non cinématographique; la mise en valeur « excessive » des plans de matte via leurs poids dans le film, la musique, le jeu des acteurs ; un montage "différent » du reste du film; une répétitivité du dispositif. Alain Resnais choisit d'exposer ainsi clairement les codes du Matte Painting, en montrant cependant, paradoxalement, à quel point il est extrêmement complexe, dans un univers " semi réaliste ", de fragmenter totalement la perspective linéaire. Ce qui expose bien, a contrario, le poids ainsi que l'importance essentielle de ce dispositif dans le cadre des Matte Paintings "traditionnels", où le peintre et le chef opérateur doivent faire correspondre trois points de vue - et même quatre, si on rajoute celui du public. Le peintre doit donc inclure le regard de la caméra qu'il va tromper dans la réalisation de 
son œuvre, de même qu'il y inclut le regard du spectateur. « C'est pourquoi si pour le sens commun au cinéma l'enregistrement est premier par rapport à la projection parce qu'en effet il l'est chronologiquement, pour le cinéma c'est la projection qui est première, principale, c'est l'image finale qui infléchit a priori tout le système. ${ }^{34}$ »

\section{Conclusion : vers une autre façon de penser les liens entre peinture et cinéma, entre perspective et cinéma}

Les rapports entre peinture et cinéma ont depuis longtemps été scrutés sous de multiples angles. « Il est semble-t-il deux façons d'aborder les relations cinéma peinture : soit l'on s'interroge sur les codes, les catégories, les conceptions communes à ces deux champs et l'on tente de les faire varier en extension et en compréhension - ainsi lorsqu'on entreprend l'étude du mouvement, de la fixité, de la lumière ou de la couleur - soit on prend comme objets des œuvres particulières : film, tableau, et l'on tente de cerner les liens qui les unissent les uns aux autres..$^{35}$ ”

Ces « deux façons » interrogent donc, d'une part, les rapports entre peinture et cinéma, d'autre part, les peintures dans le cinéma. Or, aucun ouvrage traitant de ces questions n'aborde frontalement le Matte Painting ${ }^{36}$. Comme le remarque François Albera, dans sa recension de cinq ouvrages ${ }^{37}$ aux thématiques proches sortis en 2007 :

On peut relever par ailleurs le contournement de toute question qui envisagerait les rapports effectifs, concrets de la peinture et du cinéma via les métiers du cinéma: l'importance des décorateurs, venus des Écoles de Beaux-Arts, des Arts décoratifs ou d'architecture, est ignorée, comme la place même de ces constructions plastiques qui ont pourtant compté des contributeurs de taille (Mallet-Stevens, Léger, Rodtchenko par exemple) et donné lieu à une "ligne" spécifique de l'art plastique (les grands décorateurs que furent Autant-Lara, Meerson, Bilinsky, Trauner, etc. - pour ne parler que de la France, mais la récente exposition de la Cinémathèque française sur "l’Expressionnisme" révélait un grand nombre de peintres-de-cinéma). ${ }^{38}$

Pourtant, la prise en compte du Matte Painting permet de décaler les liens entre peinture et cinéma, en proposant justement une analyse de la peinture de cinéma: peindre des plans à la manière des cinéastes. Par conséquent, analyser le Matte Painting revient à questionner à la fois la picturalité de l'image cinématographique (si une peinture peut donner l'illusion d'être un plan filmé, n'est-ce pas que le cinéma est par nature proche de la peinture ${ }^{39}$ ?), mais surtout les spécificités de cette image et de son hybridation naturelle. Car le Matte Painting emprunte ici autant aux règles de perspective issues de la peinture renaissante, qu'aux questions de cadrage, de mise en scène, de lumière, de mouvement, de montage, de durée, etc. qui sont propres au cinématographe. Le matte painter doit être capable d'analyser - au sens premier du terme - les éléments d'une image cinématographique, pour pouvoir les reproduire. La perspective «non fragment ée » que propose le Matte Painting expose ainsi, en creux, à quel point la perspective linéaire structure totalement l'illusion cinématographique, en dépit du fait qu'il est techniquement possible de faire autrement - la règle de la transparence du dispositif cinématographique reste, encore en numérique, un absolu que la non fragmentation du Matte Painting ne fait qu'appliquer à la lettre. 


\section{NOTES}

1. On trouve aussi le «matte shot ", qui peut être utilisé de manière plus large (plan avec cache) selon les contextes.

2. Pour faciliter la lecture de cet article, nous utiliserons l'expression générique Matte Painting, quel que soit le procédé technique lui ayant donné naissance, pour parler de manière globale de ce principe commun à tous les procédés.

3. METZ Christian, «Trucage et cinéma », in Essais sur la signification au cinéma (tome 2), Paris, ed. Klincksieck, 1972, p. 173-192. Cette distinction est reprise et adaptée dans Jean-Loup Bourget, « Le trompe-l'œil au cinéma », Positif $n^{\circ} 467$, janvier 2000, p. 60-64, dans un article consacré au cycle « Trompe-l'œil : l'espace illusionniste au cinéma », qui s'est tenu à l'auditorium du Louvres en septembre-octobre 1999 qui distingue le trompe-l'œil apparent (effet invisible) du trompel'œil transparent (effet imperceptible).

4. Visible sur le site des brevets américains à l'adresse : http://pdfpiw.uspto.gov/.piw? Docid=1372811\&idkey=NONE\&homeurl=http\%3A\%252F\%252Fpatft.uspto.gov\%252Fnetahtml\% 252FPTO\%252Fpatimg.htm.

5. Voir les milliers de photos mises en ligne par le collectionneur néo-zélandais Peter Cook, dans son blog hommage aux Matte Paintings pré numériques : http://nzpetesmatteshot.blogspot.fr/.

6. EVANS Chris, in Mark Cotta Vaz et Craig Barron, The Invisible Art, the Legends of Movie Matte Painting, San Francisco, Chronicle Books, 2002, p. 267, notre traduction.

7. «Interview with Albert Whitlock» par David Hutchison, Starlog, juillet 1982, p. 84, notre traduction.

8. «Ceci n'est pas un mur », dans Georges Perec, Cuchi White, L'œil ébloui, Paris, Société nouvelle des éditions du chêne, 1981, non paginé.

9. BONITZER Pascal, Décadrages, peinture et cinéma, Paris, Cahiers du cinéma, Ed. de l'Etoile, «Essais », 1985, p. 34.

10. Comme le précise Omar Calabrese, L'art du trompe-l'œil, Paris, Citadelles et Mazenod, «Les phares », 2010, p. 35 : « Le trompe-l'œil a toujours tenté de s'affranchir des limites du cadre ».

11. Pline, Histoire naturelle, Livre XXXV, §65-66. Voir aussi le commentaire de ce mythe dans Aurélie Ledoux, L'ombre d'un doute, le cinéma américain contemporain et ses trompe-l'œil, Rennes, PUR, « Le spectaculaire », 2012.

12. Ce point est surtout applicable aux trompe-l'œil de chevalet, objet le plus proche d'un Matte Painting cinématographique, et moins pour les trompe-l'œil monumentaux.

13. FARÉ Fabrice, CHEVÉ Dominique, «Les tableaux de trompe-l'œil ou la dénonciation de l'illusion, XVII ${ }^{\mathrm{e}}$ siècle ", op. cit., in Le trompe-l'œil, dirigé par Patrick Mauriès, Paris, Gallimard, 1996, p. 130.

14. MILMAN Miriam, Le trompe-l'œil, Genève, Skira, 1982, p. 36.

15. «Les tableaux de trompe-l'œil ou la dénonciation de l'illusion, XVII ${ }^{\mathrm{e}}$ siècle », Fabrice Faré, Dominique Chevé, p. 158, in Mauriès, op. cit. Voir aussi Louis Marin, « Le trompe-l'œil, un comble de la peinture ", in L'Effet trompe-l'œil dans l'art et la psychanalyse, Paris, Dunod, 1988, pp. 75-92.

16. " "Tromper les yeux", disent-ils, XIV-XV siècle ", Anne-Marie Lecoq in Mauriès, Le trompel'œil, op. cit., p. 96.

17. MILMAM Miriam, Le trompe-l'œil, op. cit., p. 7.

18. D'où la nécessité d'avoir effectivement un objet banal, connu du public, comme sujet, pour permettre un élément de comparaison avec sa représentation.

19. Qui propose d'ailleurs le concept de trompe l'intelligence au lieu de trompe-l'œil. 
20. CALABRESE, L'art du trompe-l'œil, op. cit., p. 51-52.

21. Voir le début très analysé de Body Double de Brian de Palma (1984), où le paysage en plein écran du début se révèle vite être un décor peint porté par deux personnages.

22. Ce que faisaient déjà les nombreuses retouches photographiques, combinant deux photos en une seule, comme les paysages multiples à la Gustave Le Gray (en particulier, sa collection dite des Marines, 1856-1857).

23. «Avec le plus souvent à peine cinq heures pour faire une peinture en totalité, je n'ai pas le temps de faire un travail très fini, mais j'ai compris avec l'expérience que ce n'était pas nécessaire pour obtenir le résultat», Albert Whitlock, "Special Photographic Effects for Earthquake », in George G. Turner (dir.), The ASC Treasury of Visual Effects, Hollywood, American Society of Cinematographers, 1983, p. 180, notre traduction.

24. Entretien avec Tiiu Lukk dans Super 8 Filmaker, 1977, p. 41, notre traduction.

25. Lifeboat (1940) est ainsi l'exemple ultime de ce travail, puisque le film entier a été filmé en transparence.

26. Sans le contexte historique - la guerre froide - impossible de se douter que la séquence n'a pas été réalisée dans le musée directement. Toute la séquence est en fait quasiment en studio virtuel, l'acteur, Paul Newman, n'ayant quasiment aucun repère spatial puisque le décor n'était qu'une peinture faite par Whitlock, au sommet de son art: sur les murs peints, se trouvent de tableaux peints, mais destinés à être vus comme de la peinture, alors que les murs, peints, sont eux destinés à être perçus comme des murs. "Dans ce plan, Newman n'avait aucun élément réel avec lequel interagir, et étant un acteur de la Méthode, il avait besoin d'un environnement. Donc il a demandé : "Hitch, quelle est ma motivation pour marcher tout droit?" et Hitchcock lui a répondu: "si tu ne le fais pas, tu disparaîtras derrière le matte painting" ", Joe Musso, dans Barron et Cotta Vaz, The Invisible Art..., op. cit., p. 177, notre traduction.

27. On peut aussi avoir des doutes sur la nature du plan sur l'immeuble de l'ami qui l'héberge quelques jours.

28. VIVES Jean-Marie, cité par Jean-Marc Thévenet, Images pour un film. Les décors d'Enki Bilal pour La vie est un roman d'Alain Resnais, Dargaud, Paris, 1983, p. 42.

29. "Jean-Marie Vives, peintre sur verre sur Le petit Poucet (Olivier Dahan) ", entretien avec Réjane Hamus, SFX n92, novembre/décembre 2001, p. 61.

30. VIVES Jean-Marie, dans Thévenet, Images pour un film, op. cit, p. 42.

31. Entretien inédit avec Jean-Marie Vives par Réjane Hamus-Vallée, mai 2013, Paris.

32. Expression utilisée dans Richard Rickitt, Special Effects, the History and Technique, New York, Billboard Books, 2000, p. 199, notre traduction.

33. AUMONT Jacques, L'œil interminable - Cinéma et peinture, Paris, Ed. de la différence, coll. «Les essais, ", 2007 (1989), p. 160.

34. BONITZER Pascal,, Décadrages, op. cit., p. 22.

35. JOST François," « Le picto-film » in Raymond Bellour (dir.), Cinéma et peinture, approches, Paris, Presses Universitaires de France, « Ecritures et arts contemporains », 1990, p. 109.

36. Rien ou quasi rien sur le Matte Painting dans Germain Viatte, Peinture cinéma peinture, Centre de la Vieille Charité, Marseille, 15 octobre 1989 - 14 janvier 1990, Paris, Hazan, 1989 ; Jacques Aumont, L'œil interminable, cinéma et peinture, Paris, Séguier, 1989 ; Angela Dalle Vacche, Cinema and Painting, How Art is used in Film, Austin, University of Texas Press, 1996 ; Sylvie Ramond (dir.), Impressionnisme et naissance du cinématographe, Lyon, Musée des beaux-arts de Lyon, "Varia ", 2005 ; Anne Hollander, Moving pictures, Cambridge, Harvard Univ. Press, 1991 ; Kerry Brougher (dir.), Art and Film since 1945, Hall of Mirrors, Los Angeles, The Museum of Contemporary Art, 1996. On trouve une rapide évocation des Matte Paintings de Citizen Kane et de Autant en emporte le vent dans Joëlle Moulin, Cinéma et peinture, Paris, Citadelles \& Mazenod, 2011. Seul Pascal Bonitzer, Décadrages, peinture et cinéma, Paris, Cahiers du cinéma, Ed. de l'Etoile, « Essais », 1985 consacre plusieurs pages à ce qu'il appelle le « trompe-l'œil cinématographique ». 
37. THIVAT Patricia Laure (dir.), « Peinture et cinéma. Picturalité de l'image filmée de la toile à l'écran », Ligeia, n77-80, 2007 ; Alain Bonfand, Le Cinéma saturé. Essai sur les relations de la peinture et des images en mouvement, Paris, PUF « Epiméthée ", 2007 ; Luc Vancheri, Cinéma et peinture, Paris, Armand Colin «Cinéma », 2007 ; Dominique Sipière, Alain J-J. Cohen (dir.), Les Autres Arts dans l'art du cinéma, Rennes, Presses Universitaires de Rennes, 2007 ; Berenice B. Rose (dir.), Picasso, Braque and Early Film in Cubism, New-York, Pacewildenstein, 2007.

38. ALBERA François, «Cinéma et peinture, peinture et cinéma ", 1895. Mille huit cent quatre-vingtquinze [En ligne], $54 \mid 2008$, mis en ligne le 01 février 2011, consulté le 08 juillet 2013. URL : http://1895.revues.org/2932.

39. «Par ailleurs, le film participe, dans un certain sens, à la nature de la peinture, ce qui n'est pas le cas de la scène théâtrale. L'absence des couleurs, du relief, le fait d'être délimité, d'une façon précise, par les bords de l'écran rendent le film d'autant plus accompli qu'il est dépouillé de son naturalisme. Il a toujours les caractéristiques d'une peinture à deux dimensions et, sert, en même temps, de théâtre au déroulement d'une action pleine de vie. Il est nécessaire de tenir compte de cette double nature du film, dans toute création cinématographique, qu'il s'agisse du scénario, du décor ou de la photographie», Rudolf Arnheim, «Film as Art», cité par Léon Barsacq, Le décor de film 1895-1969, Paris, Veyrier, 1985, p. 189. On pourrait même aller plus loin, et voir que «si des cinéastes s'inspirent de la peinture, c'est qu'au fond, le monde est (aussi) peinture. Révéler la beauté du monde, c'est en révéler, entre autres, la beauté picturale », Jacques Aumont, La théorie des cinéastes, Paris, Nathan, « Cinéma », 2002, p. 49.

\section{RÉSUMÉS}

Le matte painting est une technique de trucage de décor qui permet d'agrandir un espace en le prolongeant par une peinture, qu'elle soit réalisée initialement sur une vitre en verre ou par ordinateur. Cet article questionne le travail particulier de la perspective dans la réalisation de ces "trompe-l'oeil de la caméra". Il analyse en particulier le travail d'Albert Whitlock pour sa collaboration avec Alfred Hitchcock, et le peintre français Jean-Marie Vives pour La vie est un roman d'Alain Resnais.

Matte painting is a technical tricks of film décor that allows to extend a space by extending it by a painting, whether it is realized initially on a window glass or by computer. This article questions the particular work of perspective in the realization of these "trompe-l'oeil de la caméra". He analyzes in particular the work of Albert Whitlock for his collaboration with Alfred Hitchcock, and the French painter Jean-Marie Vives for La Vie est un roman, by Alain Resnais.

\section{AUTEUR}

\section{RÉJANE HAMUS-VALLÉE}

Maître de conférences habilitée à diriger des recherches au sein de l'Université d'Evry Val d'Essonne, Centre Pierre Naville, où elle dirige le Master Image et société : documentaire et sciences sociales. Elle travaille sur les effets spéciaux (Les effets spéciaux, Cahiers du cinéma/CNDP, 2004), sur les métiers du cinéma et de l'audiovisuel avec Caroline Renouard, et sur la sociologie 
visuelle et filmique (direction, Sociologie de l'image, sociologie par l'image, CinémAction, 2013). Membre du programme de recherche « Les Arts trompeurs. Machines. Magie. Médias » (Labex Arts-H2H), elle a publié en 2016 Peindre pour le cinéma. Une histoire du Matte Painting, Villeneuve d'Ascq, Presses du Septentrion, "Images et sons" et dirigé "Trucage et télévision", CIRCAV n² 25. 